\title{
The zeta-function of a $p$-adic manifold, Dwork theory for physicists
}

\author{
Philip Candelas and Xenia de la Ossa
}

In this article we review the observation, due originally to Dwork, that the $\zeta$-function of a variety, defined originally over the field with $p$ elements, is a superdeterminant. We review this observation in the context of the family of quintic 3 -folds, $\sum_{i=1}^{5} x_{i}^{5}-$ $\varphi \prod_{i=1}^{5} x_{i}=0$, and study the $\zeta$-function as a function of the parameter $\varphi$. Owing to cancellations, the superdeterminant of an infinite matrix reduces to the (ordinary) determinant of a finite matrix, $U(\varphi)$, corresponding to the action of the Frobenius map on certain cohomology groups. The $\varphi$-dependence of $U(\varphi)$ is given by a relation $U(\varphi)=E^{-1}\left(\varphi^{p}\right) U(0) E(\varphi)$ with $E(\varphi)$ a Wronskian matrix formed from the periods of the variety. The periods are defined by series that converge for $\|\varphi\|_{p}<1$. The values of $\varphi$ that are of interest are those for which $\varphi^{p}=\varphi$ so, for nonzero $\varphi$, we have $\|\varphi\|_{p}=1$. We explain how the process of $p$-adic analytic continuation applies to this case. The matrix $U(\varphi)$ breaks up into submatrices of rank 4 and rank 2 and we are able from this perspective to explain some of the observations that have been made previously by numerical calculation.

\section{Contents}

\section{Introduction}

2. Dwork's evaluation of the zeta function

2.2. Operators on a vector space 
3.2. Overconvergent series

3.3. The superdeterminant of the complex

4. Calculation of the determinants

4.1. The $4 \times 4$ determinant for $\varphi=0$

4.3. The other monomials

5. Open problems

5.1. Special geometry

5.2. The $\varphi=\infty$ limit

\section{Introduction}

A fundamental object of study for a variety over a finite field is its $\zeta$-function. Consider, for example, the one parameter family of quintic 3 -folds, $\mathcal{M}_{\varphi}$, defined by the vanishing of the polynomial

$$
P(x, \varphi)=\sum_{i=1}^{5} x_{i}^{5}-\varphi \prod_{i=1}^{5} x_{i}
$$

which is the family of varieties that will largely occupy us here. If $\varphi$ takes values in $\mathbb{F}_{p}$, the field with $p$ elements, and the variety is considered as a subvariety of $\mathbb{F}_{p} \mathbb{P}^{4}$, then one can compute $N_{1}(\varphi)$ the number of solutions to (1.1). More generally one can take $\varphi \in \mathbb{F}_{p}$ and the coordinates $x_{j} \in \mathbb{F}_{p^{m}}$ and denote the number of solutions by $N_{m}(\varphi)$. The $\zeta$-function is defined as 
a generating function for these numbers

$$
\zeta(\varphi, T)=\exp \left\{\sum_{m=1}^{\infty} N_{m}(\varphi) \frac{T^{m}}{m}\right\} .
$$

The form of the $\zeta$-function as a function of $T$ is greatly restricted by the Weil conjectures [1], which have since been proved. One of these conjectures, proved by Dwork [2], states that the $\zeta$-function is a rational function of $T$. The proof proceeds by showing that the $\zeta$-function is a ratio of products of determinants that of the form $\operatorname{det}(1-U(\varphi) T)$ for certain finite matrices $U(\varphi)$ that are independent of $T$.

Dwork showed $[3,4]$ that the $\zeta$-function is a superdeterminant (though this was not stated in this language) of a matrix that expresses the action of the Frobenius map on a differential complex associated to the variety. The Frobenius map is important in what follows so we pause to review it here.

Consider again the variety defined by the equation $P(x, \varphi)=0$ where we take the coefficients of $P$ to take values in $\mathbb{F}_{p}$ (in our case this is just the statement $\varphi \in \mathbb{F}_{p}$ ) but we allow $x$ to take values in a larger field such as the algebraic closure of $\mathbb{F}_{p}$ or, as we shall want to do later, in $\mathbb{C}_{p}$ which is the completion of the algebraic closure of $\mathbb{Q}_{p}$, the field of $p$-adic numbers. Now, since $P(x, \varphi)=0$ we have $P(x, \varphi)^{p}=0$. Hence

$$
P\left(x^{p}, \varphi\right)=0, \text { with } x^{p}=\left(x_{1}^{p}, x_{2}^{p}, x_{3}^{p}, x_{4}^{p}, x_{5}^{p}\right)
$$

and where we have used the fact that $\varphi^{p}=\varphi$. In this way, we see that the Frobenius map

$$
\operatorname{Fr}(x)=x^{p}
$$

is an automorphism of $\mathcal{M}_{\varphi}$. The fixed points of this map are the points for which $x_{j}^{p}=x_{j}, j=1, \ldots, 5$ and this is precisely the condition that $x$ be defined over $\mathbb{F}_{p}$. Thus $N_{1}$ is the number of fixed points of the Frobenius map. ${ }^{1}$ The approach of Dwork to the problem of calculating the numbers $N_{m}(\varphi)$ was to define a cohomology theory adapted to the $p$-adic context and then to use an appropriate analog of the Lefshetz fixed point theorem that applies to varieties defined over $\mathbb{C}$. This permits the Euler number of the fixed point set of an automorphism to be calculated as the trace of a matrix representing the action of the automorphism on the cohomology of the variety.

\footnotetext{
${ }^{1}$ The rigidity theorem (for an accessible account see, for example, [5]) states that the Frobenius map generates the full Galois group, so one can equally say that $N_{1}$ is the number of fixed points of the Galois action.
} 
We work through this analysis here in the context of the quintic 3-fold in a way that we hope is straightforward. The quantities that are familiar in the complex case, particularly when the variety can be embedded in a toric variety, duly make their appearance. In particular, the Newton polytope of the variety and the cone of monomials over the Newton polyhedron play an important role as do the periods of the variety and the Picard-Fuchs equation. An intriguing difference that we do not resolve is that, in the present calculation, the periods appear as infinite series rather than in the truncated forms of $[6]$.

The reader is warned at the outset that, in attempting to present a reasonably self-contained account, we work through material that is by now very classical. There are reviews of the work of Dwork, such as those by Katz $[7,8]$ that have themselves become classic references. To quote from these references would allow us to shorten this account considerably though at the expense of being self-contained.

Dwork represents the (inverse of) the action of the Frobenius map by a matrix $U(\varphi)$ that is given in a $p$-adic neighborhood of $\varphi=0$ by the expression

$$
U(\varphi)=E^{-1}\left(\varphi^{p}\right) U(0) E(\varphi)
$$

where $E$ is the Wronskian matrix formed from the periods of the variety. The periods are defined by series, and the series converge on the disk $\|\varphi\|_{p}<1$. If the series were to converge also for the Teichmüller points for which $\varphi^{p}=\varphi$ then the $\zeta$-function, which is calculated in terms of determinants of the form $\operatorname{det}(1-U(\varphi) T)$ would not depend on $\varphi$. In fact, the series diverge for $\|\varphi\|_{p}=1$ and the matrix $U(\varphi)$ has to be defined via a process of $p$-adic analytic continuation. This leads to a matrix $U(\varphi)$ which is defined on the Teichmüller points but which is no longer of the form (1.2).

In [9], the $\zeta$-function was calculated for the family (1.1). This was done numerically in virtue of the fact that the numbers of points $N_{m}(\varphi)$ can be computed rapidly in terms of the periods of $\mathcal{M}_{\varphi}$ (at least for $p$ not too large and for the first few values of $m$ ). This was sufficient to suggest that the $\zeta$-function factorizes in the form

$$
\zeta(\varphi, T)=\frac{R_{\mathbf{1}}(\varphi, T) R_{A}\left(\varphi, p^{\rho} T^{\rho}\right)^{20 / \rho} R_{B}\left(\varphi, p^{\rho} T^{\rho}\right)^{30 / \rho}}{(1-T)(1-p T)\left(1-p^{2} T\right)\left(1-p^{3} T\right)}
$$

the interesting point here is the form of the numerator since the form of the denominator is standard. In this expression, $\rho(=1,2$ or 4$)$ is the smallest integer such that $5 \mid p^{\rho}-1$ and the quantities $R_{\mathbf{1}}, R_{A}, R_{B}$ are quartics in 
their second argument. For example, $R_{\mathbf{1}}$ takes the form

$$
R_{\mathbf{1}}(\varphi, T)=1+a(\varphi) T+b(\varphi) p T^{2}+a(\varphi) p^{3} T^{3}+p^{6} T^{4}
$$

with $a(\varphi)$ and $b(\varphi)$ integers that depend on $\varphi$.

The organization of this paper is as follows: in $\S 2$ we review the theory of the Dwork character and show how this relates to an operator on a vector space. The vector space in question is that of power series in the coordinates $x_{j}$. The $\zeta$-function for $\mathcal{M}_{\varphi}$ is the superdeterminant of a matrix acting on a complex of forms of degree up to 5 . The matrices are, at this stage, infinite matrices acting on the infinite-dimensional space of power series. By defining a suitable covariant derivative, as we do in $\S 3$, the eigenvalues of the supermatrix are seen to cancel in the superdeterminant apart from a finite number that correspond to the action of the supermatrix on finite-dimensional cohomology groups. Furthermore, the superdeterminant corresponding to these cohomology groups itself decomposes into a product of factors that can be identified with the quartics $R_{1}, R_{A}, R_{B}$ that appear in (1.3). The explicit computation of these determinants is the subject of $\S 4$.

Our interest in this formalism arises, in part, from a desire to study the arithmetic properties of the moduli spaces of $p$-adic Calabi-Yau varieties. We hope to return to this topic elsewhere, particularly in regard to arithmetic special geometry and arithmetic properties of the attractor mechanism. It is likely that the both the attractor varieties and the parameters to which they correspond will have interesting arithmetic properties. We are interested also in the fact that Dwork's analysis manifests processes that are familiar, in other contexts, to physicists. In particular, the process of reducing infinite-dimensional Hilbert spaces to a finite-dimensional supersymmetric subspace is familiar in the context of topological field theory. In this context, there is a standard way of passing to the states and operators of the supersymmetric subspace with the operators having the property that they commute or anticommute with the supersymmetry generators. In the context of Dwork theory, the cohomology spaces that are the analogs of the supersymmetric subspace, have parameters and Dwork finds an interesting and essential use for operators that do not commute with the cohomology generators since these can map between spaces corresponding to different parameter values. Our aim in this article is to explain these points. The reader is warned that we do not aspire to complete mathematical rigor, particularly with regard to processes such as the reduction of the infinitedimensional superdeterminants to the finite-dimensional superdeterminants of matrices that act on the cohomology spaces, though we do explain how 
this reduction comes about. The detailed $p$-adic analysis is given in the original papers of Dwork.

One final disclaimer is in order. The fact that the zeta-function of a variety may be understood in terms of the action of the Frobenius automorphism on suitably defined cohomology groups is central to the Weil Conjectures and as such has been central to much of modern number theory. These observations have been refined and improved and, given sufficient background, there are other, more modern, ways of proceeding from a variety to its $\zeta$-function [10-12]. The algorithmic complexity of the computation of the $\zeta$-function of a variety is also of interest in relation to cryptography. The issue, in this context, is the rapid computation of the $\zeta$-function for a general variety for fields $\mathbb{F}_{p^{r}}$ with $r$ large and $p$ small. These considerations do not concern us here though we note that, as a matter of practical calculation, the methods of [6] work well in the regime of small $r$ for $p<500$, say.

After this work was complete we became aware of the recent paper of Kloosterman [13] which has substancial overlap with the present work.

\section{Dwork's evaluation of the zeta function}

\subsection{Review of Dwork's character}

We begin by reviewing the properties of Dwork's character $\Theta: \mathbb{F}_{p} \rightarrow \mathbb{C}_{p}^{*}$; a full account may be found in $[14,15]$. In order to define $\Theta$, we define first a function $\mathfrak{F}$ as a power series

$$
\mathfrak{F}(X)=\exp \left(\pi\left(X-X^{p}\right)\right)=\sum_{n=0}^{\infty} c_{n}(\pi X)^{n},
$$

where $\pi$ is a number in $\mathbb{C}_{p}$ such that $\pi^{p-1}=-p$. The exponential is understood as given by the usual power series and the resulting power series in $X$ defines $\mathfrak{F}$ in the first instance. By differentiating $\mathfrak{F}$ we find

$$
\frac{1}{\pi} \frac{d \mathfrak{F}}{d X}=\left(1+(\pi X)^{p-1}\right) \mathfrak{F}
$$

and it is easy to show from this relation that the series for $\mathfrak{F}$ has the form shown and that the coefficients satisfy the recurrence $n c_{n}=c_{n-1}+c_{n-p}$ with $c_{n}=0$ for $n<0$ and $c_{0}=1$. Now it is an essential fact that the series on the right of (2.1) converges in the disk $\|X\|_{p} \leq 1+\epsilon$ for some fixed positive $\epsilon$. The exponential series $\exp (\pi Y)$ converges, for $p$-adic $Y$, in the disk $\|Y\|_{p}<1$ and the $X$-disk that ensures $\left\|X-X^{p}\right\|_{p}<1$ is the disk $\|X\|_{p}<1$. We now 
give an improved definition for $\mathfrak{F}(X)$ as the sum of the series in (2.1) valid throughout the disk where this series converges

$$
\mathfrak{F}(X)=\sum_{n=0}^{\infty} c_{n}(\pi X)^{n} ; \quad\|X\|_{p} \leq 1+\epsilon
$$

So defined $\mathfrak{F}(X)$ exists and is $p$-adic analytic in the disk $\|X\|_{p} \leq 1+\epsilon$. On the smaller disk $\|X\|_{p}<1$, we have $\mathfrak{F}(X)=\exp \left(\pi\left(X-X^{p}\right)\right)$. We shall need also to evaluate $\mathfrak{F}(X)$ for $\|X\|_{p}=1$. For such $X$ the series converges, however, as we shall see shortly, it does not converge to $\exp \left(\pi\left(X-X^{p}\right)\right)$.

The character $\Theta: \mathbb{F}_{p} \rightarrow \mathbb{C}_{p}^{*}$, of order $p$, is defined by

$$
\Theta(x)=\mathfrak{F}(\operatorname{Teich}(x))
$$

The Teichmüller representative $X=$ Teich $(x)$ corresponds to the embedding of $\mathbb{F}_{p}^{*}$ in $\mathbb{C}_{p}^{*}$ as a multiplicative group. We think of $x$ as an integer in the range $0 \leq x \leq p-1$ and then we define $\operatorname{Teich}(x)$ as a limit

$$
\operatorname{Teich}(x)=\lim _{n \rightarrow \infty} x^{p^{n}}
$$

which converges in the $p$-adic sense. Thus Teich $(x)=x+\mathcal{O}(p)$ and $\operatorname{Teich}(x)$ satisfies the equation

$$
\operatorname{Teich}(x)^{p}=\operatorname{Teich}(x)
$$

In virtue of our previous discussion, however, we are prepared for the fact that even though this last relation holds nevertheless $\Theta(x) \neq \exp (0)=1$. In fact, we see that

$$
\Theta(1)=1+\pi+\mathcal{O}\left(\pi^{2}\right)
$$

and since $\|\pi\|_{p}<1$ it follows that $\Theta(1) \neq 1$. If, however, $X^{p}=X$ then

$$
\mathfrak{F}(X)^{p}=\exp \left(p \pi X-p \pi X^{p}\right)=1
$$

since the presence of the $p$ in the exponent ensures the convergence of the series. In particular, $\Theta(1)$ is a $p$ th root of unity and since $\Theta(x)=$ $1+\pi$ Teich $(x)+\mathcal{O}\left(\pi^{2}\right)$ we see also that $\Theta(x)=\Theta(1)^{\text {Teich }(x)}=\Theta(1)^{x}$. 
It follows from the definition of the Teichmüller representative that for $x, y \in \mathbb{F}_{p}$

$$
\operatorname{Teich}(x+y)=\operatorname{Teich}(x)+\operatorname{Teich}(y)+p Z
$$

for some $Z \in \mathbb{Z}_{p}$. From this we see that $\Theta$ is a nontrivial additive character, that is

$$
\Theta(x+y)=\Theta(x) \Theta(y),
$$

and moreover is of order $p$ since $\Theta(p x)=1$.

Dwork also adapted this construction to give a character of order $p$, $\Theta_{r}: \mathbb{F}_{p^{r}} \rightarrow \mathbb{C}_{p}^{*}$

$$
\Theta_{r}(x)=\Theta(\operatorname{tr}(x)),
$$

where $\operatorname{tr}: \mathbb{F}_{p^{r}} \rightarrow \mathbb{F}_{p}$ is the trace map

$$
\operatorname{tr}(x)=x+x^{p}+x^{p^{2}}+\cdots+x^{p^{r-1}} .
$$

Now the limit Teich $(x)=\lim _{n \rightarrow \infty} x^{q^{n}}$ exists also for $x \in \mathbb{F}_{q}$ though Teich $(x)$, for $x \neq 0$, is now a unit (has unit norm) of $\mathbb{C}_{p}$ but is not, in general ${ }^{2}$, in $\mathbb{Z}_{p}$. We have

$$
\operatorname{Teich}(\operatorname{tr}(x))=\sum_{\ell=0}^{r-1} \operatorname{Teich}^{p^{\ell}}(x)+p Z
$$

with $Z$ an integer of $\mathbb{C}_{p}$. It follows that

$$
\Theta_{r}(x)=\Theta(1)^{\sum_{\ell=0}^{r-1} \operatorname{Teich}^{p^{\ell}}(x)} .
$$

Note, however, that we cannot, in general, write the right-hand side of this relation as the product

$$
\prod_{\ell=0}^{r-1} \Theta(1)^{\operatorname{Teich}^{p^{\ell}}(x)}
$$

since the Teich ${ }^{p^{\ell}}(x)$ are not, in general, in $\mathbb{Z}_{p}$. Dwork showed nevertheless that $\Theta_{r}$ has the remarkable splitting property

$$
\Theta_{r}(x)=\prod_{\ell=0}^{r-1} \Theta\left(x^{p^{\ell}}\right) .
$$

\footnotetext{
${ }^{2}$ Recall that $\mathbb{F}_{q}$ is an extension of degree $r$ over $\mathbb{F}_{p}$. That is, $\mathbb{F}_{q}=\mathbb{F}_{p}(\alpha)$ with $\alpha$, the root of an irreducible monic polynomial of degree $r$ with coefficients in $\mathbb{F}_{p}$. Thus for $x \in \mathbb{F}_{q}$ the Teichmüller representative will have the form $\operatorname{Teich}(x)=\sum_{k=0}^{r-1} b_{k} \alpha^{k}$ with coefficients in $\mathbb{Q}_{p}$ (in fact in $\mathbb{Z}_{p}$ ) but the root $\alpha$ is not in $\mathbb{Q}_{p}$.
} 
The characters in the product make sense through the series (2.2) for $\|X\|_{p} \leq$ $1+\epsilon$ even if $X$ is in $\mathbb{C}_{p}$ rather than $\mathbb{Q}_{p}$.

To show the utility of Dwork's character, let $P(X) \in \mathbb{F}_{p}\left[X^{1}, X^{2}, \ldots, X^{n}\right]$ be a homogeneous polynomial of degree $d$ and let $\nu_{r}^{*}$ denote the number of $x=\left(x^{1}, \ldots, x^{n}\right) \in \mathbb{F}_{p^{r}}^{n}$, with no component zero, of solutions to the equation $P(x)=0$, that is

$$
\nu_{r}^{*}=\#\left\{x \in\left(\mathbb{F}_{p^{r}}^{*}\right)^{n} \mid P(x)=0\right\}
$$

Then in virtue of the relation

$$
\sum_{y \in \mathbb{F}_{p^{r}}} \Theta_{r}(y P(x))= \begin{cases}p^{r}, & \text { if } P(x)=0 \\ 0, & \text { otherwise }\end{cases}
$$

we have

$$
p^{r} \nu_{r}^{*}=\left(p^{r}-1\right)^{n}+\sum_{(y, x) \in\left(\mathbb{F}_{p^{r}}^{*}\right)^{n+1}} \Theta_{r}(y P(x))
$$

where the first term on the right arises from splitting off the $y=0$ contribution to the sum.

It is convenient to set $x^{0}=y$ and to write

$$
W(x)=x^{0} P\left(x^{1}, \ldots, x^{n}\right)
$$

We will have need of some notation relating to polynomials and series. For monomials, we employ a multi-index notation and write $X^{\mathbf{v}}=\prod_{\alpha=0}^{n}\left(X^{\alpha}\right)^{v_{\alpha}}$. We shall refer to $\sum_{j=1}^{n} v_{j}$ as the degree of $X^{\mathbf{v}}$ ignoring $v_{0}$. The set of all $X^{\mathbf{v}}$ of degree $\ell d$ with $v_{0}=\ell, \ell=0,1, \ldots$, define an $n$-dimensional lattice $\Lambda$. Within this lattice the monomials $X^{\mathbf{v}}$ that arise with nonzero coefficient in $W(X)$ define a polyhedron $\Delta$. We shall have need also of the cone $K \subset \Lambda$ subtended from $\mathbf{v}=0$ by $\Delta$. For the case of $W$, a generic cubic and $n=3$ this is illustrated in figure 1. With these conventions we can write $W(x)=$ $\sum_{\mathbf{v} \in \Delta} w_{\mathbf{v}} x^{\mathbf{v}}$ and we shall understand $W(X)$ for $p$-adic $X$ to be given by

$$
W(X)=\sum_{\mathbf{v} \in \Delta} W_{\mathbf{v}} X^{\mathbf{v}}, \text { with } W_{\mathbf{v}}=\operatorname{Teich}\left(w_{\mathbf{v}}\right)
$$




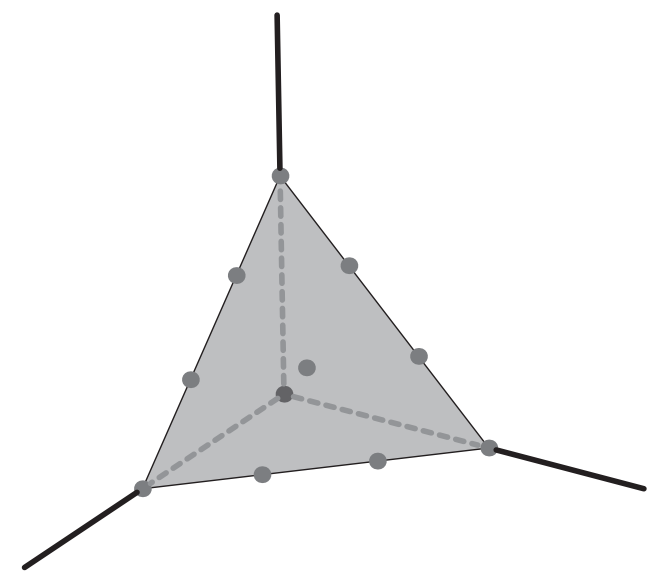

Figure 1: The cone $K$ and polyhedron $\Delta$ for the generic cubic.

We have explained how the character $\Theta(W(x))$ is related to the series

$$
\mathfrak{F}(W(X))=\exp \left(\pi\left(W(X)-W(X)^{p}\right)\right)
$$

We wish now to show also that $\Theta(W(x))=\mathfrak{G}(\operatorname{Teich}(x))$ for $\mathfrak{G}(X)$ the function defined by the power series

$$
\mathfrak{G}(X)=\exp \left(\pi\left(W(X)-W\left(X^{p}\right)\right)\right)=\sum_{\mathbf{v} \in K} G_{\mathbf{v}} X^{\mathbf{v}}
$$

In other words, we claim that the series on the right converges on the polydisk $\left\|X^{\alpha}\right\|_{p} \leq 1$ though again it does not converge, when $\|X\|_{p}=1$, to $\exp \left(\pi\left(W(X)-W\left(X^{p}\right)\right)\right)$. To see that $\Theta(W(x))=\mathfrak{G}($ Teich $(x))$ note that

$$
\begin{aligned}
\Theta(W(x)) & =\prod_{\mathbf{v} \in \Delta} \Theta\left(w_{\mathbf{v}} x^{\mathbf{v}}\right) \\
& =\lim _{X \rightarrow \operatorname{Teich}(x)} \prod_{\mathbf{v} \in \Delta} \exp \left[\pi\left(W_{\mathbf{v}} X^{\mathbf{v}}-W_{\mathbf{v}} X^{p \mathbf{v}}\right)\right] \\
& =\lim _{X \rightarrow \operatorname{Teich}(x)} \exp \left[\pi\left(W(X)-W\left(X^{p}\right)\right)\right] \\
& =\lim _{X \rightarrow \operatorname{Teich}(x)} \mathfrak{G}(X) .
\end{aligned}
$$


Similar relations apply also to the functions $\Theta\left(W(x)^{p^{\ell}}\right)$ and in this way we see that our expression (2.4) can be rewritten in the form

$$
p^{r} \nu_{r}^{*}=\left(p^{r}-1\right)^{n}+\sum_{\left(X^{\alpha}\right)^{p^{r}-1}=1} \mathfrak{G}(X) \mathfrak{G}\left(X^{p}\right) \cdots \mathfrak{G}\left(X^{p^{r-1}}\right) .
$$

\subsection{Operators on a vector space}

One of Dwork's great insights was to regard the computation of the $\zeta$-function as a sequence of operations on a vector space in a way that bears an intriguing similarity to the formalism of quantum mechanics. The analog of a Hilbert space is here a ring $\mathcal{H}$ of power series of the form $\Phi(X)=$ $\sum_{\mathbf{v} \in K} \Phi_{\mathbf{v}} X^{\mathbf{v}}$, where the powers $X^{\mathbf{v}}$ that occur lie in the cone $K$ and the coefficients $\Phi_{\mathbf{v}}$ are required to decrease such that $\left\|\Phi_{\mathbf{v}}\right\|_{p}<\|\pi\|_{p}^{\operatorname{deg} \mathbf{v}}$ as $\operatorname{deg} \mathbf{v} \rightarrow \infty$.

A basis of states is provided by the monomials $\left\{X^{\mathbf{v}}\right\}_{\mathbf{v} \in K}$ which, following the usage of quantum mechanics, we can think of abstractly as states $|\mathbf{v}\rangle$. There is no notion of Hermitian conjugation, however, we may nevertheless define a dual basis $\langle\mathbf{v}|$ by requiring

$$
\langle\mathbf{u} \mid \mathbf{v}\rangle= \begin{cases}1, & \text { if } \mathbf{u}=\mathbf{v} \\ 0, & \text { otherwise }\end{cases}
$$

In $\mathcal{H}$, series $\Psi(X)$ are both states and operators, since a series $\Psi(X)$ maps a state $\Phi(X)$ to the state $\Psi(X) \Phi(X)$.

Dwork made important use of an operator $\mathfrak{A}_{q}: \mathcal{H} \rightarrow \mathcal{H}$, that seems to have been first introduced by Adkin, and which may be regarded as an inverse to the Frobenius map

$$
\mathfrak{A}_{q}\left(X^{\mathbf{v}}\right)= \begin{cases}X^{\mathbf{v} / q}, & \text { if } q \mid \mathbf{v} \\ 0, & \text { otherwise }\end{cases}
$$

Equivalently the action of $\mathfrak{A}_{q}$ on a series $\Phi(X)=\sum_{\mathbf{v} \in K} \Phi_{\mathbf{v}} X^{\mathbf{v}}$ can be written

$$
\mathfrak{A}_{q} \Phi(X)=\sum_{\mathbf{v} \in K} \Phi_{q \mathbf{v}} X^{\mathbf{v}}
$$

Dwork uses the notation $\psi_{q}$ for this map and this notation is common in the literature. This, however, would be confusing in an account that attempts to draw analogies with quantum mechanics owing to the established use of $\psi$ in the latter context to denote state vectors. 
It is straightforward to establish the operator identities:

$$
\begin{array}{ll}
\text { 1. } & \left(\mathfrak{A}_{q}\right)^{r}=\mathfrak{A}_{q^{r}} \\
\text { 2. } & \mathfrak{A}_{q} \Phi\left(X^{q}\right)=\Phi(X) \mathfrak{A}_{q} \\
\text { 3. } & \mathfrak{A}_{q^{r}} \Phi(X) \Phi\left(X^{q}\right) \cdots \Phi\left(X^{q^{r-1}}\right)=\left(\mathfrak{A}_{q} \Phi(X)\right)^{r} .
\end{array}
$$

We shall have need also of the matrix elements of operators of the form $\mathfrak{A}_{q} \Phi(X)$. These can be written in terms of the coefficients of $\Phi$

$$
\left\langle\mathbf{u}\left|\mathfrak{A}_{q} \Phi(X)\right| \mathbf{v}\right\rangle=\Phi_{q \mathbf{u}-\mathbf{v}}
$$

where it is understood that $\Phi_{\mathbf{w}}=0$ for $\mathbf{w} \notin K$.

Consider now the effect of summing $\Phi(X)$ over the images of $\left(\mathbb{F}_{q}^{*}\right)^{n+1}$. We will see that this is related to the trace of the matrix $\mathfrak{A}_{q} \Phi(X)$

$$
\sum_{\left(X^{\alpha}\right)^{q-1}=1} \Phi(X)=(q-1)^{n+1} \sum_{\mathbf{v} \in K} \Phi_{(q-1) \mathbf{v}}=(q-1)^{n+1} \operatorname{Tr}\left(\mathfrak{A}_{q} \Phi(X)\right) .
$$

We wish to apply this identity to the sum that arises in (2.6) in relation to the calculation of $\nu^{*}$. In virtue of the identities above we find

$$
\begin{aligned}
& \quad \sum_{\left(X^{\alpha}\right)^{p^{r}-1}=1} \mathfrak{G}(X) \mathfrak{G}\left(X^{p}\right) \cdots \mathfrak{G}\left(X^{p^{r-1}}\right) \\
& =(q-1)^{n+1} \operatorname{Tr}\left(\mathfrak{A}_{p^{r}} \mathfrak{G}(X) \mathfrak{G}\left(X^{p}\right) \cdots \mathfrak{G}\left(X^{p^{r-1}}\right)\right) \\
& =(q-1)^{n+1} \operatorname{Tr}\left(\left(\mathfrak{A}_{p} \mathfrak{G}(X)\right)^{r}\right) .
\end{aligned}
$$

We may also write

$$
\mathfrak{U}=\mathfrak{A}_{p} \mathfrak{G}(X)=\mathrm{e}^{-\pi W(X)} \mathfrak{A}_{p} \mathrm{e}^{\pi W(X)} .
$$

This last expression is useful for purposes of manipulation, however, it must be borne in mind that, while $\mathfrak{G}(X) \in \mathcal{H}$, the series $\mathrm{e}^{ \pm \pi W(X)}$ are not in $\mathcal{H}$. Thus it is not the case that $\operatorname{Tr}(\mathfrak{U})$ is $\operatorname{Tr}\left(\mathfrak{A}_{p}\right)$, for example.

Using our identities in (2.6) we have

$$
\nu_{r}^{*}=\frac{\left(p^{r}-1\right)^{n}}{p^{r}}+\frac{\left(p^{r}-1\right)^{n+1}}{p^{r}} \operatorname{Tr}\left(\mathfrak{U}^{r}\right) .
$$


We wish to use this expression to compute a function $Z^{*}(T)$ that is closely allied to the $\zeta$-function

$$
Z^{*}(T)=\exp \left\{\sum_{r=1}^{\infty} \frac{\nu_{r}^{*} T^{r}}{r}\right\}
$$

In order to do this we note that

$$
\exp \left\{\sum_{r=1}^{\infty} \operatorname{Tr}\left(\mathfrak{U}^{r}\right) \frac{T^{r}}{r}\right\}=\exp \{-\operatorname{Tr} \log (1-\mathfrak{U} T)\}=\operatorname{det}(1-\mathfrak{U} T)^{-1} .
$$

If we now expand the powers of $\left(p^{r}-1\right)$ in $(2.7)$ by the binomial theorem we find

$$
Z^{*}(T)=\prod_{j=0}^{n}\left(1-p^{j-1} T\right)^{(-1)^{(n-j+1)}}\left(\begin{array}{c}
n \\
j
\end{array}\right) \prod_{k=0}^{n+1} \operatorname{det}\left(1-p^{k-1} T \mathfrak{U}\right)^{(-1)^{(n-k)}\left(\begin{array}{c}
n+1 \\
k
\end{array}\right)}
$$

At this stage the determinants that appear in this expression are determinants of infinite matrices, however, Dwork has proved that $Z^{*}$ is a rational function of $T$. This is a consequence of the fact that, owing to the alternating sign of the exponents in the last equation, determinants appear both in the numerator and the denominator and there is cancellation between all but a finite number of eigenvalues (note that the matrices are all diagonal if $\mathfrak{U}$ is). We are interested in how this cancellation comes about. This is a story about cohomology to which we now turn.

\section{Dwork cohomology}

\subsection{Exterior and covariant derivatives}

Following Monsky and Washnitzer (for a good review, see [16] the original reference is [17]), we define an analog of an exterior derivative that acts on power series. The "differential forms" require for their definition formal symbols $d X^{\alpha}$. We start by defining logarithmic derivatives and differentials

$$
D_{\alpha}=X^{\alpha} \frac{\partial}{\partial X^{\alpha}}, \quad \xi^{\alpha}=\frac{d X^{\alpha}}{X^{\alpha}}
$$


In order to commute with $\mathfrak{U}=\mathrm{e}^{-\pi W(X)} \mathfrak{A}_{p} \mathrm{e}^{\pi W(X)}$, we take our exterior derivative to be

$$
\mathfrak{D}=\mathrm{e}^{-\pi W(X)} d X^{\alpha} \frac{\partial}{\partial X^{\alpha}} \mathrm{e}^{\pi W(X)}=\mathrm{e}^{-\pi W(X)} \xi^{a} D_{\alpha} \mathrm{e}^{\pi W(X)}
$$

where, here and in the following, a summation convention applies to repeated indices. The $\xi^{\alpha}$ are taken to anticommute among themselves so we shall simply write $\xi^{\alpha_{1}} \cdots \xi^{\alpha_{k}}$ instead of $\bigwedge_{i=1}^{k} \xi^{\alpha_{i}}$. Spaces, $\mathcal{H}_{k}$, of $k$-forms are defined to be

$$
\mathcal{H}_{k}=\left\{\Phi_{\alpha_{1} \alpha_{2} \cdots \alpha_{k}}(X) \xi^{\alpha_{1}} \xi^{\alpha_{2}} \cdots \xi^{\alpha_{k}} \mid \Phi_{\alpha_{1} \alpha_{2} \cdots \alpha_{k}}(X)=\Phi_{\left[\alpha_{1} \alpha_{2} \cdots \alpha_{k}\right]}(X) \in \mathcal{H}\right\},
$$

where $\Phi_{\left[\alpha_{1} \alpha_{2} \cdots \alpha_{k}\right]}$ denotes the skew part. We obtain in this way a chain complex

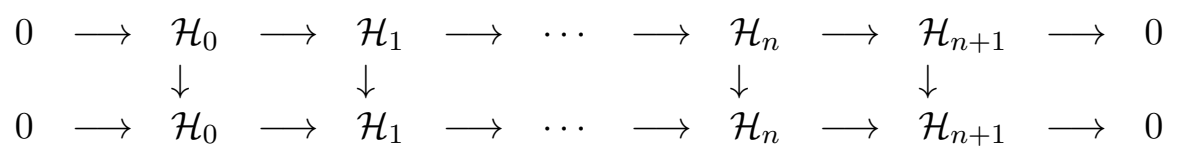

with the horizontal maps being $\mathfrak{D}$ and the vertical maps $\mathfrak{U}$. Note that each $\mathcal{H}_{k}$ is isomorphic, as a vector space, to $\bigwedge^{k} \mathcal{H}_{1}$.

Owing to the fact that $\xi^{\alpha}=d \log \left(X^{\alpha}\right)$ and the effect of $\mathfrak{A}_{p}$ is, in effect, to replace $X^{\alpha}$ by $\left(X^{\alpha}\right)^{1 / p}$ we define

$$
\mathfrak{A}_{p}\left(\xi^{\alpha}\right)=\frac{1}{p} \xi^{\alpha} \quad \text { hence also } \quad \mathfrak{U}\left(\xi^{\alpha}\right)=\frac{1}{p} \xi^{\alpha} .
$$

With this convention it is easy to check that $\mathfrak{D}$ and $\mathfrak{U}$ commute. It is sufficient to check this for a form $\mathrm{e}^{-\pi W} X^{\mathbf{v}} \xi^{\beta_{1}} \cdots \xi^{\beta_{k}}$

$$
\begin{array}{ccc}
\mathrm{e}^{-\pi W} X^{\mathbf{v}} \xi^{\beta_{1}} \cdots \xi^{\beta_{k}} & \stackrel{\mathfrak{D}}{\longrightarrow} & \mathrm{e}^{-\pi W} X^{\mathbf{v}} v_{\alpha} \xi^{\alpha} \xi^{\beta_{1}} \cdots \xi^{\beta_{k}} \\
\mathfrak{U} \downarrow & & \mathfrak{U} \downarrow \\
\frac{1}{p^{k}} \mathrm{e}^{-\pi W} \mathfrak{A}_{p}\left(X^{\mathbf{v}}\right) \xi^{\beta_{1}} \cdots \xi^{\beta_{k}} & \stackrel{\mathfrak{D}}{\longrightarrow} & \frac{1}{p^{k+1}} \mathrm{e}^{-\pi W} \mathfrak{A}_{p}\left(X^{\mathbf{v}}\right) v_{\alpha} \xi^{\alpha} \xi^{\beta_{1}} \cdots \xi^{\beta_{k}} .
\end{array}
$$

Having been defined so as to commute with $\mathfrak{U}$ the operator $\mathfrak{D}$ should be regarded as a covariant derivative. This being so, a comment is in order regarding the manner in which $\mathfrak{D}$ respects the Leibnitz rule. If we write $\xi^{\alpha} D_{\alpha}=D$ then we have

$$
\mathfrak{D}(\phi \Psi)=\phi \mathfrak{D} \Psi+(D \phi) \Psi
$$

which is appropriate if we regard $\Psi$ as a vector and $\phi$ as an operator. We learn that $\mathfrak{D}$ acts differently on vectors and operators. Note, however, the 
necessary consistency property that the derivative of a vector $\Phi=\phi \Psi$ does not depend on the decomposition of the product into its factors.

\subsection{Overconvergent series}

In order to have a well-behaved cohomology theory, it is necessary to place convergence conditions on the series that arise in the differential forms. A first thought is that one should require the series to converge on the polydisks $\left\|X^{\alpha}\right\|_{p} \leq 1$. This, however, is inadequate as we see already from the following standard one-dimensional example. Consider the 1-form

$$
\omega=\sum_{n=0}^{\infty} p^{n} x^{p^{n}-1} d x=d\left(\sum_{n=0}^{\infty} x^{p^{n}}\right) .
$$

Owing to the fact that $\omega$ is a 1 -form and the space is one-dimensional, we have $d \omega=0$. There is, however, no 0 -form $\eta$ such that $\omega=d \eta$, since the series on the right does not converge on the unit disk owing to the fact that it does not converge for $\|x\|_{p}=1$. Thus if we merely require convergence on the polydisks $\left\|X^{\alpha}\right\|_{p} \leq 1$, then there is a failure of the Poincaré lemma: that, for a contractible space, a form that is closed is also exact. It was shown by Dwork in [3] that this difficulty may be overcome by requiring that the series converge on polydisks of radius $1+\delta$ for some $\delta>0$. These are referred to as overconvergent series. For a certain choice of $\delta$ the set of overconvergent series is the ring

$$
\mathcal{H}=\left\{\sum_{\mathbf{v} \in K} a_{\mathbf{v}} \pi^{\operatorname{deg}(\mathbf{v})} X^{\mathbf{v}} \mid a_{\mathbf{v}} \rightarrow 0 \text { as } \operatorname{deg}(\mathbf{v}) \rightarrow \infty\right\}
$$

In the cited work it is shown, moreover, that for this choice of $\mathcal{H}$ the only nontrivial cohomology occurs in $\mathcal{H}_{0}$ and $\mathcal{H}_{n+1}$.

\subsection{The superdeterminant of the complex}

The superdeterminant of the operator $\left(1-p^{n} T \mathfrak{U}\right)$ of the complex $\mathcal{H}_{\bullet}$ is

$$
\operatorname{sdet}_{\mathcal{H}}\left(1-p^{n} T \mathfrak{U}\right)=\prod_{\ell=0}^{n+1} \operatorname{det}_{\mathcal{H}_{\ell}}\left(1-p^{n} T \mathfrak{U}\right)^{(-1)^{\ell}}
$$

and we wish to show now that this superdeterminant is precisely the product of determinants that arises in $Z^{*}(T)$. Referring back to $(2.8)$ we see that 
the latter expression contains the product

$$
\left[\prod_{\ell=0}^{n+1} \operatorname{det}_{\mathcal{H}_{0}}\left(1-p^{n-\ell} T \mathfrak{U}\right)^{(-1)^{\ell}\left(\begin{array}{c}
n+1 \\
\ell
\end{array}\right)}\right]^{-1}
$$

where we have written $k=n+1-\ell$ for the index in the product and we note that, in this context, the determinants refer to matrices that act on $\mathcal{H}_{0}$.

An eigenfunction $\Phi(X) \in \mathcal{H}_{0}$ of $\mathfrak{U}$, that has eigenvalue $\mu$, contributes a factor $\left(1-p^{n} \mu T\right)$ to $\operatorname{det}_{\mathcal{H}_{0}}\left(1-p^{n} T \mathfrak{U}\right)$. This eigenfunction also gives rise to $\left(\begin{array}{c}n+1 \\ \ell\end{array}\right)$ eigenforms in each $\mathcal{H}_{\ell}$ of the form $\Phi(X) \xi^{\alpha_{1}} \cdots \xi^{\alpha_{\ell}}$ each satisfying

$$
p^{n} \mathfrak{U}\left(\Phi(X) \xi^{\alpha_{1}} \cdots \xi^{\alpha_{\ell}}\right)=p^{n-\ell} \mu \Phi(X) \xi^{\alpha_{1}} \cdots \xi^{\alpha_{\ell}}
$$

Thus $\Phi(X)$ contributes a factor $\prod_{\ell=0}^{n+1}\left(1-p^{n-\ell} \mu T\right)^{(-1)^{\ell}\left(\begin{array}{c}n+1 \\ \ell\end{array}\right)}$ to the superdeterminant. Furthermore, it is immediate that every eigenform $\Psi=\Psi_{\alpha_{1} \cdots \alpha_{\ell}}$ $\xi^{\alpha_{1}} \cdots \xi^{\alpha_{\ell}}$ of $\mathfrak{U}$ has coefficients $\Psi_{\alpha_{1} \cdots \alpha_{\ell}}$ in $\mathcal{H}_{0}$ that are eigenfunctions of $\mathfrak{U}$. Thus all the eigenforms are of the form $\Phi(X) \xi^{\alpha_{1}} \cdots \xi^{\alpha_{\ell}}$ and we see that the alternating product in the square brackets in (3.1) is precisely the superdeterminant of the complex.

$$
\operatorname{sdet}_{\mathcal{H}}\left(1-p^{n} T \mathfrak{U}\right)=\prod_{\ell=0}^{n+1} \operatorname{det}_{\mathcal{H}_{0}}\left(1-p^{n-\ell} T \mathfrak{U}\right)^{(-1)^{\ell}\left(\begin{array}{c}
n+1 \\
\ell
\end{array}\right)}
$$

If now $\Psi^{(\ell)}(X) \in \mathcal{H}_{\ell}$ is a $\ell$-form which is an eigenform of $\mathfrak{U}$ with eigenvalue $\mu$ then, since $\mathfrak{D}$ and $\mathfrak{U}$ commute we have also

$$
\mathfrak{U}\left(\mathfrak{D} \Psi^{(\ell)}(X)\right)=\mu \mathfrak{D} \Psi^{(\ell)}(X)
$$

and we see that if $\mathfrak{D} \Psi^{(\ell)}(X)$ is nonzero it is a $(\ell+1)$-form with the same eigenvalue as $\Psi^{(\ell)}(X)$. The contribution of such eigenforms cancels from the superdeterminant and in this way, we see that the superdeterminant reduces to a superdeterminant on the $\mathfrak{D}$-cohomology of the complex.

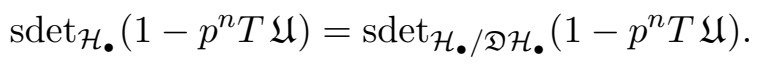

It is important that the Dwork cohomology groups are finite-dimensional. This is a nontrivial fact for which a proof, valid for an arbitrary smooth variety, was given in 1997 by Berthelot [18]. 
Now that the states have become forms we shall define the inner product $\langle\Phi \mid \Psi\rangle$ to be the constant term in the expansion of the highest order form, that is the coefficient of $\xi^{0} \xi^{1} \cdots \xi^{n}$. Given that the only nontrivial cohomology occurs in $\mathcal{H}_{0}$ and $\mathcal{H}_{n+1}$ we shall principally be concerned with computing products $\langle\phi \mid \Psi\rangle$ between zero-forms $\langle\phi|=\phi^{*}(X)$ and $(n+1)$-forms $|\Psi\rangle=\psi(X) \xi^{0} \xi^{1} \cdots \xi^{n}$. When this is the case we may write

$$
\langle\phi \mid \Psi\rangle=(\phi, \psi)
$$

with the inner product on the right denoting the inner product defined previously for zero-forms.

Now if $|\chi\rangle=\frac{1}{n !} \chi^{\alpha}(X) \epsilon_{\alpha \beta_{1} \beta_{2} \cdots \beta_{n}} \xi^{\beta_{1}} \xi^{\beta_{2}} \cdots \xi^{\beta_{n}}$, where $\epsilon_{\alpha \beta_{1} \beta_{2} \cdots \beta_{n}}$ denotes the permutation symbol, is an $n$-form then $\mathfrak{D}|\chi\rangle=\mathfrak{D}_{\alpha} \chi^{\alpha} \xi^{0} \xi^{1} \cdots \xi^{n}$ and we see that in cohomology

$$
\psi(X) \simeq \psi(X)+\mathfrak{D}_{\alpha} \chi^{\alpha}(X)
$$

In order for our inner products to respect this equivalence, the dual states must satisfy

$$
\left(\phi, \mathfrak{D}_{\alpha} \chi^{\alpha}\right)=0
$$

for all $\chi^{\alpha}$.

Let $\gamma_{-}$denote the operator that projects onto the space of dual states

$$
\gamma_{-} X^{\mathbf{v}}= \begin{cases}X^{\mathbf{v}}, & \text { if }-\mathbf{v} \in K, \operatorname{deg}(\mathbf{v}) \neq 0 \\ 0, & \text { otherwise }\end{cases}
$$

and let

$$
\mathfrak{D}_{\alpha}^{*}=-\gamma_{-} \mathrm{e}^{\pi W(X)} D_{\alpha} \mathrm{e}^{-\pi W(X)}
$$

then a little thought shows that

$$
\left(\phi, \mathfrak{D}_{\alpha} \psi\right)=\left(\mathfrak{D}_{\alpha}^{*} \phi, \psi\right)
$$

for all $\phi$ and $\psi$. Thus (3.2) requires the condition $\mathfrak{D}_{\alpha}^{*} \phi^{*}(X)=0$ be imposed on the allowed dual states. 


\section{Calculation of the determinants}

In this section, we will verify that the $\zeta$-function for the quintic takes the form

$$
\zeta(\varphi, T)=\frac{\operatorname{det}(1-U(\varphi) T / p)}{(1-T)(1-p T)\left(1-p^{2} T\right)\left(1-p^{3} T\right)}
$$

with $\mathfrak{U}(\varphi)$ acting on certain cohomology groups that we will find. The cohomology groups are in correspondence with monomials $x^{\mathbf{v}}$ with $\operatorname{deg}(\mathbf{v})=0,5,10,15,20$ and we will find that the $\operatorname{determinant} \operatorname{det}(1-U T / p)$ breaks up naturally into a part that depends on $\varphi$ and a part that is independent of $\varphi$. The $\varphi$-dependent part has the correct dimension 204 and corresponds to monomials $x^{\mathbf{v}}$ with $\operatorname{deg}(\mathbf{v})=0,5,10,15,20$ whose exponent vectors $\mathbf{v}$ have no component zero. With the matrix $U$ restricted to this subspace the $\zeta$-function takes the form (4.1). We believe that it is straightforward to deduce this form by performing an iteration based on (2.8) but we have not performed the calculation.

We anticipate from [6] and [9] that the monomials of degree $5 \ell, 0 \leq \ell \leq 5$, will play a special role. Among these, there are 126 monomials of degree 5 . The fifth powers, $x_{i}^{5}$, and the twenty monomials whose exponents are permutations of $(2,1,1,1,0)$ turn out to be trivial in cohomology. In the case of the monomials which are permutations of $(2,1,1,1,0)$ these are related to monomials that are permutations of $(4,1,0,0,0)$ and will not need to be counted separately. We are left with 101 quintic monomials. The monomial

$$
Q \stackrel{\text { def }}{=} x_{0} x_{1} x_{2} x_{3} x_{4} x_{5}
$$

plays a special role, and there are 100 others that we denote by $x^{\mathbf{v}}$. We anticipate also that the determinants which are in principle of size $204 \times 204$ break up into a $4 \times 4$ block, corresponding to a basis $\left\{Q, Q^{2}, Q^{3}, Q^{4}\right\}$, and 100 blocks of size $2 \times 2$ corresponding to bases $\left\{x^{\mathbf{v}}, Q x^{\mathbf{v}}\right\}$, one for each of the quintic monomials $x^{\mathbf{v}}$. We begin by considering the case $\varphi=0$.

\subsection{The $4 \times 4$ determinant for $\varphi=0$}

When $\varphi=0$ the operator whose determinant we wish to compute is

$$
\mathfrak{U}(0)=\mathfrak{A}_{p} \prod_{i=1}^{5} \mathfrak{F}\left(X_{0} X_{i}^{5}\right) .
$$


Consider the effect of this operator on a basis $|\ell\rangle=(-\pi Q)^{\ell}$.

$$
\mathfrak{U}(0)|\ell\rangle=(-1)^{\ell} \mathfrak{A}_{p} \sum_{n_{1}, \ldots, n_{5} \geq 0}\left(\prod_{i=1}^{5} c_{n_{i}}\right) \pi^{\sum_{i} n_{i}+\ell} X_{0}^{\sum_{i} n_{i}+\ell} \prod_{i} X_{i}^{5 n_{i}+\ell}
$$

The terms in the sum that survive the effect of $\mathfrak{A}_{p}$ are those for which

$$
5 n_{i}+\ell \equiv 0 \bmod p, \quad i=1, \ldots, 5 .
$$

Note that these conditions imply that $\sum_{i} n_{i}+\ell \equiv 0, \bmod p$ on summation over $i$. To proceed, it is useful to define integers $1<a<p-1$ and $1 \leq b \leq 4$ such that $a$ is the smallest positive integer that represents $-1 / 5$ in $\mathbb{F}_{p}$ and $b$ satisfies

$$
5 a+1=b p .
$$

The integers $a$ and $b$ depend on the value of $p \bmod 5$, and are given in table 1 .

Equation (4.3) is solved by writing

$$
n_{i}=n(\ell)+k_{i} p
$$

where

$$
n(\ell)=a \ell-p\left[\frac{a \ell}{p}\right]
$$

is the smallest positive representative of $a \ell \bmod p$. Since $5 n(\ell)+\ell \equiv$ $0 \bmod p$ there is an integer $0 \leq r(\ell) \leq 4$ such that

$$
5 n(\ell)+\ell=r(\ell) p .
$$

A little thought reveals that, for $p \neq 5, r(\ell)$ has the property that it is the smallest positive residue of $b \ell \bmod 5$

$$
r(\ell)=b \ell-5\left[\frac{b \ell}{5}\right]
$$

Returning to (4.2) we see that

$$
\mathfrak{U}(0)|\ell\rangle=(-1)^{\ell} p^{r} \prod_{i=1}^{5}\left(\sum_{k_{i}=0}^{\infty} c_{k_{i} p+n} p^{k_{i}}\left(-\pi X_{0} X_{i}^{5}\right)^{k_{i}}\right)|r(\ell)\rangle .
$$


Now for any state $|\chi\rangle$, we have the following identity that holds in cohomology

$$
\mathfrak{D}_{i}|\chi\rangle=\left(D_{i}+5 \pi X_{0} X_{i}^{5}-\pi \varphi Q\right)|\chi\rangle=0
$$

or equivalently

$$
\left(-\pi X_{0} X_{i}^{5}\right)|\chi\rangle=\mathrm{e}^{\pi \varphi Q} \frac{D_{i}}{5} \mathrm{e}^{-\pi \varphi Q}|\chi\rangle
$$

Where we defer setting $\varphi=0$ for the present. Now it follows from this last relation that

$$
\left(-\pi X_{0} X_{i}^{5}\right)^{m}|\chi\rangle=\mathrm{e}^{\pi \varphi Q}\left(\frac{D_{i}}{5}\right)_{m} \mathrm{e}^{-\pi \varphi Q}|\chi\rangle .
$$

A word of caution is warranted here. Note that the right-hand side of this last relation contains the Pochhammer symbol

$$
\left(\frac{D_{i}}{5}\right)_{m}=\left(\frac{D_{i}}{5}\right) \cdot\left(\frac{D_{i}}{5}+1\right) \ldots\left(\frac{D_{i}}{5}+m-1\right)
$$

not the power $\left(D_{i} / 5\right)^{m}$. This is so, because we must unwrap the powers of $X$ from the outside in order to use (4.4). Thus we have

$$
\begin{aligned}
\left(-\pi X_{0} X_{i}^{5}\right)^{m}|\Psi\rangle & =-\pi X_{0} X_{i}^{5}\left(\left(-\pi X_{0} X_{i}^{5}\right)^{m-1}|\Psi\rangle\right) \\
& =\mathrm{e}^{\pi \varphi Q} \frac{D_{i}}{5} \mathrm{e}^{-\pi \varphi Q}\left(\left(-\pi X_{0} X_{i}^{5}\right)^{m-1}|\Psi\rangle\right) \\
& =\left(-\pi X_{0} X_{i}^{5}\right)^{m-1} \mathrm{e}^{\pi \varphi Q}\left(\frac{D_{i}}{5}+m-1\right) \mathrm{e}^{-\pi \varphi Q}|\Psi\rangle
\end{aligned}
$$

To naively unwrap the power from the inside would be wrong since the equalities are true in cohomology, so $|\Psi\rangle=|\Phi\rangle$ means, of course, that $|\Psi\rangle-$ $|\Phi\rangle=\mathfrak{D}|\chi\rangle$ for some $|\chi\rangle$. The formalism has the awkward feature that we can have $|\Psi\rangle=|\Phi\rangle$, in this sense, yet $f(X)|\Psi\rangle \neq f(X)|\Phi\rangle$, in general, since $f(X) \mathfrak{D}|\chi\rangle$ is not necessarily a derivative. Worse still, it follows that we can have $|\Psi\rangle=0$ but $f(X)|\Psi\rangle \neq 0$. We could avoid this awkwardness by restricting the allowed operators to be those that commute with $\mathfrak{D}$, however, that would leave us with a very small class of operators; in the present case, we would be limited to just the constants. These are indeed the operators that map $\mathcal{H} / \mathfrak{D}(\varphi) \mathcal{H}$ to itself, for fixed $\varphi$. An essential aim in the following is, however, to study how the cohomology varies with $\varphi$. It is precisely because the operators we use do not commute with $\mathfrak{D}$ that they can map 
between spaces corresponding to different values of $\varphi$. For example we shall see shortly that

$$
\mathrm{e}^{-\pi \varphi Q}: \mathcal{H} / \mathfrak{D}(\varphi) \longrightarrow \mathcal{H} / \mathfrak{D}(0)
$$

It is better therefore to proceed carefully with these caveats in mind.

Returning to our calculation, we take note of a relation for the $p$-adic $\Gamma$-function [15] established recently by Robert [19]

$$
\Gamma_{p}(p z-a)=\sum_{k=0}^{\infty} c_{k p+a} p^{k}(z)_{k} ; \quad 0 \leq a \leq p-1, z \in \mathbb{Z}_{p}
$$

and we see that our expressions simplify considerably yielding

$$
\mathfrak{U}(0)|\ell\rangle=(-1)^{\ell} p^{r} \Gamma_{p}^{5}\left(\frac{\ell}{5}\right)|r(\ell)\rangle
$$

where in writing this last relation we have used the fact that $\mathrm{pr} / 5-n=$ $\ell / 5$. The matrix $\langle k|\mathfrak{U}(0)| \ell\rangle$ is of size $5 \times 5$, has one entry in each row and column and $\langle 0|\mathfrak{U}(0)| 0\rangle=1$. The determinant of the $4 \times 4$ block with $1 \leq k$, $l \leq 4$, after the replacement $t \rightarrow t / p$ yields the the factor $R_{1}(t, 0)$ of the zeta function of $[9]$.

Recall that $r(\ell)$ is the reduction of $b \ell \bmod 5$. We see from table 1 , that $b^{\rho} \equiv 1 \bmod 5$ precisely when $p^{\rho} \equiv 1 \bmod 5$, in fact, for $p \neq 2,5$, we have that $b$ is the smallest positive integer that is $1 / p \bmod 5$. For $\rho=1$, that is $5 \mid p-1$, we have $b=1$ and $r(\ell)=\ell$ so the matrix is diagonal and one easily verifies the corresponding quartics $R_{\mathbf{1}}(t, 0)$ given in [9]. For $\rho=2$ we have $\mathfrak{U}(0)^{2}|\ell\rangle=-p^{5}|\ell\rangle, 1 \leq \ell \leq 4$, leading to $R_{1}(t, 0)=\left(1+p^{3} t^{2}\right)^{2}$ in these cases. For $\rho=4$ the states form a cycle of length four and $\mathfrak{U}(0)^{4}|\ell\rangle=$ $-p^{10}|\ell\rangle, 1 \leq \ell \leq 4$ leading to $R_{\mathbf{1}}(t, 0)=1+p^{6} t^{4}$.

Table 1: The quantities $a$ and $b$ as functions of $p$.

\begin{tabular}{ccccc}
\hline$p \bmod 5$ & $a$ & $b$ & relation & $\rho$ \\
\hline 1 & $\frac{p-1}{5}$ & 1 & $b=1$ & 1 \\
2 & $\frac{3 p-1}{5}$ & 3 & $b^{4} \equiv 1$ & 4 \\
3 & $\frac{2 p-1}{5}$ & 2 & $b^{4} \equiv 1$ & 4 \\
4 & $\frac{4 p-1}{5}$ & 4 & $b^{2} \equiv 1$ & 2 \\
\hline
\end{tabular}


For $\varphi=0$ it is straightforward to see that a basis of solutions to the equations $\mathfrak{D}_{\alpha}^{*} \Phi^{*}(X)=0$ is provided by the dual states

$$
\left\langle\mathbf{u}\left|=X^{-\mathbf{u}} \prod_{i=1}^{5} \sum_{r_{i}=0}^{\infty} \frac{\left(\frac{u_{i}}{5}\right)_{r_{i}}}{\left(-\pi X_{0} X_{i}^{5}\right)^{r_{i}}} ; \quad 0 \leq u_{i} \leq 4,5\right| \operatorname{deg}(\mathbf{u})\right.
$$

and that these states are dual to the states $X^{\mathbf{v}}$.

\subsection{Variation of structure}

We turn now to the case $\varphi \neq 0$ and the operator

$$
\begin{aligned}
\mathfrak{U}(\varphi) & =\mathfrak{A}_{p} \exp \left(\pi\left(W(X)-W(X)^{p}\right)\right) \\
& =\mathfrak{A}_{p} \prod_{i=1}^{5} \mathfrak{F}\left(X_{0} X_{i}^{5}\right) \mathfrak{F}(-\varphi Q) \\
& =\mathrm{e}^{\pi \varphi^{p} Q} \mathfrak{U}(0) \mathrm{e}^{-\pi \varphi Q} .
\end{aligned}
$$

In order to evaluate the matrix for the operator $\mathfrak{U}(\varphi)$ explicitly we first take $\|\varphi\|_{p}<1$, for which the operators $\mathrm{e}^{-\pi \varphi Q}$ and $\mathrm{e}^{\pi \varphi^{p} Q}$ preserve $\mathcal{H}$, and we will later continue $\mathfrak{U}(\varphi)$ to $\|\varphi\|_{p}=1$. In our discussion of cohomology in the previous section, it was implicit that the parameters of $W$ satisfied relations analogous to $\varphi^{p}=\varphi$. Now we have $\|\varphi\|_{p}<1$ so a term $\varphi^{p} Q^{p}$ arises in $W(X)^{p}$ and this leads to the presence of $\varphi^{p}$ in the expression for $\mathfrak{U}(\varphi)$.

We wish to study how the matrix $\mathfrak{U}(\varphi)$ acts on the cohomology groups $\mathcal{H} / \mathfrak{D}$. To emphasize the dependence of the states in the quotient space on the parameter we append a $\varphi$ to the states. Thus the state $|\mathbf{v}\rangle_{\varphi}$, for example, denotes the equivalence class of $X^{\mathbf{v}}$ in $\mathcal{H} / \mathfrak{D}$. The parameter dependence arises because the states now correspond to equivalence classes and the $\mathfrak{D}$-operator depends on $\varphi$. We write $\mathfrak{D}(\varphi)$ to emphasize this dependence; setting $W=W_{0}-\varphi Q$ we have

$$
\mathfrak{D}(\varphi)=\mathrm{e}^{\pi \varphi Q} \mathrm{e}^{-\pi W_{0}} D \mathrm{e}^{\pi W_{0}} \mathrm{e}^{-\pi \varphi Q}=\mathrm{e}^{\pi \varphi Q} \mathfrak{D}(0) \mathrm{e}^{-\pi \varphi Q}
$$

Now, by writing out the derivative explicitly, we see that

$$
\frac{1}{5} \mathfrak{D}_{i}(\varphi)|\Psi\rangle=\left(\pi X_{0} X_{i}^{5}+\mathrm{e}^{\pi \varphi Q} \frac{D_{i}}{5} \mathrm{e}^{-\pi \varphi Q}\right)|\Psi\rangle
$$


As a relation on $\mathcal{H} / \mathfrak{D}(\varphi)$ this becomes

$$
\left(-\pi X_{0} X_{i}^{5}\right)|\Psi\rangle_{\varphi}=\mathrm{e}^{\pi \varphi Q} \frac{D_{i}}{5} \mathrm{e}^{-\pi \varphi Q}|\Psi\rangle_{\varphi}
$$

The corresponding relation on $\mathcal{H} / \mathfrak{D}(0)$ is

$$
\left(-\pi X_{0} X_{i}^{5}\right)|\Psi\rangle_{0}=\frac{D_{i}}{5}|\Psi\rangle_{0}
$$

In virtue of $(4.5)$, we also have $\mathrm{e}^{-\pi \varphi Q} \mathfrak{D}(\varphi)=\mathfrak{D}(0) \mathrm{e}^{-\pi \varphi Q}$. It follows that

$$
\mathrm{e}^{-\pi \varphi Q}(|\Psi\rangle+\mathfrak{D}(\varphi)|\chi\rangle)=\left|\mathrm{e}^{-\pi \varphi Q} \Psi\right\rangle+\mathfrak{D}(0)\left|\mathrm{e}^{-\pi \varphi Q} \chi\right\rangle
$$

From this and an analogous relation involving $\mathrm{e}^{\pi \varphi Q}$ we learn that

$$
\mathrm{e}^{-\pi \varphi Q}: \mathcal{H} / \mathfrak{D}(\varphi) \rightarrow \mathcal{H} / \mathfrak{D}(0) \quad \text { and } \quad \mathrm{e}^{\pi \varphi Q}: \mathcal{H} / \mathfrak{D}(0) \rightarrow \mathcal{H} / \mathfrak{D}(\varphi)
$$

A restatement of the above is that

$$
\mathrm{e}^{-\pi \varphi Q}|\Psi\rangle_{\varphi}=\left|\mathrm{e}^{-\pi \varphi Q} \Psi\right\rangle_{0} \quad \text { and } \quad \mathrm{e}^{\pi \varphi Q}|\Phi\rangle_{0}=\left|\mathrm{e}^{\pi \varphi Q} \Phi\right\rangle_{\varphi}
$$

We find it convenient to build the exponential factors into the notation and write

$$
|\Psi ; \varphi\rangle \stackrel{\text { def }}{=} \mathrm{e}^{-\pi \varphi Q}|\Psi\rangle_{\varphi} \quad \text { and } \quad|\Psi ; 0\rangle \stackrel{\text { def }}{=}|\Psi\rangle_{0}
$$

We apply these considerations to the operator $\mathfrak{U}(\varphi)$ which is a composition of maps between the following spaces.

$$
\begin{array}{ccc}
\mathcal{H} / \mathfrak{D}(\varphi) & \stackrel{\mathrm{e}^{-\pi \varphi Q}}{\longrightarrow} & \mathcal{H} / \mathfrak{D}(0) \\
\mathfrak{U}(\varphi) \downarrow & & \downarrow \mathfrak{U}(0) \\
\mathcal{H} / \mathfrak{D}\left(\varphi^{p}\right) & \stackrel{\mathrm{e}^{\pi \varphi^{p} Q}}{\longleftarrow} & \mathcal{H} / \mathfrak{D}(0)
\end{array}
$$

We will understand the matrix for $\mathfrak{U}(\varphi)$ to have components

$$
U_{j \ell}(\varphi)={ }_{\varphi^{p}}\left\langle j\left|\mathrm{e}^{\pi \varphi^{p} Q} \mathfrak{U}(0) \mathrm{e}^{-\pi \varphi Q}\right| \ell\right\rangle_{\varphi}=\left\langle j ; \varphi^{p}|\mathfrak{U}(0)| \ell ; \varphi\right\rangle .
$$

The operator $\mathfrak{U}(\varphi)$ maps between spaces that are in general different but which become the same when $\varphi^{p}=\varphi$. Now for the state on the right of this 
last matrix element we can write

$$
\begin{aligned}
|\ell ; \varphi\rangle & =\left|\mathrm{e}^{-\pi \varphi Q}(-\pi Q)^{\ell} ; 0\right\rangle \\
& =\sum_{n=0}^{\infty} \frac{\varphi^{n-\ell}}{(n-\ell) !}|n ; 0\rangle \\
& =\sum_{j=0}^{4} \sum_{m=0}^{\infty} \frac{\varphi^{5 m+j-\ell}}{(5 m+j-\ell) !}|5 m+j ; 0\rangle \\
& =\sum_{j=0}^{4} \sum_{m=0}^{\infty} \frac{\varphi^{5 m+j-\ell}}{(5 m+j-\ell) !}\left(\frac{j}{5}\right)_{m}^{5}|j ; 0\rangle
\end{aligned}
$$

where the third line follows from breaking up the $n$-sum by writing $n=$ $5 m+j, 0 \leq j \leq 4$, and the fourth by noting that

$$
(-\pi Q)^{5 m}|\Psi ; 0\rangle=\prod_{i=1}^{5}\left(-\pi X_{0} X_{i}^{5}\right)^{m}|\Psi ; 0\rangle=\prod_{i=1}^{5}\left(\frac{D_{i}}{5}\right)_{m}|\Psi ; 0\rangle .
$$

In this way, we see that

$$
|\ell ; \varphi\rangle=\sum_{j=0}^{4}|j ; 0\rangle E_{j \ell}(\varphi)
$$

with

$$
E_{j \ell}(\varphi)=\sum_{m=0}^{\infty} \frac{\varphi^{5 m+j-\ell}}{(5 m+j-\ell) !}\left(\frac{j}{5}\right)_{m}^{5}
$$

It follows also that

$$
\left\langle j ; \varphi^{p}\right|=\left\langle(-\pi Q)^{j} \mathrm{e}^{-\pi \varphi^{p} Q} ; 0\right|=\sum_{k=0}^{4} E_{j k}^{-1}\left(\varphi^{p}\right)\langle k ; 0|
$$

and hence we arrive at the expression

$$
U(\varphi)=E^{-1}\left(\varphi^{p}\right) U(0) E(\varphi)
$$

We are seeking to calculate the $5 \times 5$ determinant $\operatorname{det}(1-U(\varphi) T / p)$. The matrices $E^{-1}, U(0)$ and $E$ and hence also $U(\varphi)$ all have a first row $(1,0,0,0,0)$. Thus apart from a factor of $(1-T / p)$ the $5 \times 5$ determinant 
reduces to a $4 \times 4$ determinant of the same form but with the matrix indices running over the range $1 \leq j, \ell \leq 4$. We shall abuse notation by denoting these reduced matrices by the same symbols as previously.

The matrix $E_{j \ell}, 1 \leq j, \ell \leq 4$ is a Wronskian matrix as we see by noting that

$$
E_{j \ell}(\varphi)=\left(\frac{d}{d \varphi}\right)^{\ell-1} E_{j 1}(\varphi)
$$

and that $\varphi E_{j 1}$ satisfies the Picard-Fuchs ${ }^{3}$ equation

$$
\mathcal{L}_{\varphi}\left(\varphi E_{j 1}(\varphi)\right)=0, \quad \text { with } \mathcal{L}_{\varphi}=\left(\frac{\varphi}{5}\right)^{5} \delta_{\varphi}^{4}-\prod_{i=1}^{4}\left(\delta_{\varphi}-i\right)
$$

with $\delta_{\varphi}=\varphi \frac{d}{d \varphi}$. Being a Wronskian the determinant of $E$ has a simple form

$$
\operatorname{det} E=\frac{1}{\left(1-(\varphi / 5)^{5}\right)^{2}}
$$

The components of the matrices $E(\varphi)$ and $E^{-1}(\varphi)$ are power series in $\varphi$ with coefficients that are $p$-adic fractions. However, it appears to be the case, on the basis of numerical experiment, that $U(\varphi)$ is a matrix whose components are power series with coefficients that are $p$-adic integers. Integrality of these coefficients would follow it; one could show that the basis we use "comes from" crystalline cohomology. One may well be able to do this using results from $[20, \S 3]$. In any event equation (4.10) provides a power series expansion for $U(\varphi)$. This series converges for $\|\varphi\|_{p}<1$ but not for $\|\varphi\|_{p}=1$ since the coefficients do not tend to zero. It has, however, been shown by Lauder [10] (see especially the Appendix) that the offending coefficients can, however, be summed to a rational function. For the present case, numerical experiment suggests that we may write

$$
U(\operatorname{Teich}(5) \psi)=\left(\frac{1-\psi^{5}}{1-\psi^{5 p^{n}}}\right) \widetilde{U}_{n}(\operatorname{Teich}(5) \psi)+\mathcal{O}\left(p^{N}\right)
$$

with $\widetilde{U}_{n}(\varphi)$ a polynomial in $\varphi$ of order approximately $5 p^{n}$ and $N>n$. For example, if $p=3,7$ or 11 and $n=3$ then $N=8$ (in fact for $p=3$ we have $N=9)$. Thus we may compute $\widetilde{U}(\varphi)$ to arbitrary $p$-adic precision by taking

\footnotetext{
${ }^{3}$ The Picard-Fuchs operator that we give here is related to the corresponding Picard-Fuchs operator, $\mathcal{L}$, of $[6]$ by $\mathcal{L}_{\varphi}=\frac{\varphi^{5}}{5} \mathcal{L}$.
} 
$n$ sufficiently large and then setting $\psi^{p}=\psi$ in $\widetilde{U}_{n}$. It is a consequence of the relation (4.11) that the limit

$$
\widetilde{U}(\varphi)=\lim _{n \rightarrow \infty} \widetilde{U}_{n}(\varphi)
$$

exists and is a convergent series for $\|\varphi\|_{p}=1$. Note also that if we understand the rational function as a limit we have

$$
\frac{1-\psi^{5}}{1-\psi^{5 p^{n}}}= \begin{cases}1 ; & \psi=\operatorname{Teich}(u), u \in \mathbb{F}_{p}, u^{5} \neq 1 \\ \frac{1}{p^{n}} ; & \psi^{5}=1 .\end{cases}
$$

We find that $p^{n} \mid \widetilde{U}_{n}(\varphi)$ for $\psi^{5}=1$, so that we may use (4.11) to compute $U$ even for these values. Take now $\varphi=\operatorname{Teich}(5 u), u \in \mathbb{F}_{p}$, with $u^{5} \neq 1$ then we have

$$
\operatorname{det}(1-\tilde{U}(\varphi) T / p)=1+\tilde{a}(\varphi) T+\tilde{b}(\varphi) p T^{2}+\tilde{a}(\varphi) p^{3} T^{3}+p^{6} T^{4}
$$

with the coefficients most easily calculated as

$$
\tilde{a}=-\frac{1}{p} \operatorname{tr} \widetilde{U} \quad \text { and } \quad \tilde{b}=\frac{1}{2 p^{3}}\left((\operatorname{tr} \widetilde{U})^{2}-\operatorname{tr}\left(\widetilde{U}^{2}\right)\right) .
$$

In this way, we recover the values of the coefficients given in the tables of [9].

Having observed that the matrix $U$ may be evaluated for $\|\varphi\|_{p}=1$ by resumming an infinite series it follows that $U$ may be calculated by the simpler expedient of choosing a sufficiently large integer $s$, expanding the matrices $E(\varphi)$ and $E^{-1}\left(\varphi^{p}\right)$ to $p^{s}$ and $p^{s-1}$ terms, respectively, and evaluating on the Teichmüller point in each of these sums. We have remarked above that neither the power series for $E$ nor the power series for $U$ converge when $\|\varphi\|_{p}=1$. Nevertheless the procedure just outlined provides a convergent sequence of approximants to $U$ as $s \rightarrow \infty$. The convergence of the series for $\widetilde{U}(\varphi)$ may well be a consequence of the overconvergence discovered by Dwork [21] for hypergeometric functions with rational parameters that satisfy certain conditions.

\subsection{The other monomials}

By applying a procedure closely analogous to that of $\S 3.1$ we may calculate the matrix $U$ in a basis corresponding to the monomials $|\mathbf{v}\rangle=(-\pi)^{v_{0}} X^{\mathbf{v}}$. 
We find that

$$
\mathfrak{U}(0)|\mathbf{v}\rangle=(-1)^{v_{0}} p^{r_{0}} \prod_{i=1}^{5} \Gamma_{p}\left(\frac{v_{i}}{5}\right)|\mathbf{r}\rangle
$$

where $r_{i}$ is the smallest positive residue of $b v_{i} \bmod 5$ and $b, 1 \leq b \leq 4$, is again such that $b p=1 \bmod 5$. It is straightforward also to find the relations analogous to (4.8) and (4.9). Let $X^{\tilde{\mathbf{v}}}$ be the monomial resulting from the extraction of as many powers of $Q$ from $X^{\mathbf{v}}$ as possible, so that $X^{\mathbf{v}}=Q^{\ell} X^{\tilde{\mathbf{v}}}$ and at least one component of $\tilde{\mathbf{v}}$ is zero. We have

$$
\begin{aligned}
\left|\mathrm{e}^{-\pi \varphi Q}(-\pi)^{v_{0}} X^{\mathbf{v}} ; 0\right\rangle & =\left|\mathrm{e}^{-\pi \varphi Q}(-\pi)^{\ell+\tilde{v}_{0}} Q^{\ell} X^{\tilde{\mathbf{v}}} ; 0\right\rangle \\
& =\sum_{j=0}^{4}|j \mathbf{1}+\tilde{\mathbf{v}} ; 0\rangle E_{\tilde{\mathbf{v}}, j, l}(\varphi)
\end{aligned}
$$

with

$$
E_{\tilde{\mathbf{v}}, j, l}(\varphi)=\sum_{m=0}^{\infty} \frac{\varphi^{5 m+j-\ell}}{(5 m+j-\ell) !} \prod_{i=1}^{5}\left(\frac{j+\tilde{v}_{i}}{5}\right)_{m}
$$

To apply this result, consider the family of monomials obtained from a given monomial $X^{\mathbf{v}}$ by multiplying successively by powers of $Q$ and then reducing the exponents mod 5 . The families that descend in this way from our representative quintics are displayed in table 2.

Table 2: The four five-dimensional cohomology groups with the corresponding representatives of the two-dimensional subgroups distinguished by $\mathrm{a} *$.

\begin{tabular}{cccr}
\hline$(1,4,0,0,0)$ & $(3,2,0,0,0)$ & $(3,1,1,0,0)$ & $(2,2,1,0,0)$ \\
\hline$(1,4,0,0,0)$ & $(3,2,0,0,0)$ & $(3,1,1,0,0)$ & $(2,2,1,0,0)$ \\
$(2,0,1,1,1)$ & $*(4,3,1,1,1)$ & $*(4,2,2,1,1)$ & $*(3,3,2,1,1)$ \\
$*(3,1,2,2,2)$ & $(0,4,2,2,2)$ & $(0,3,3,2,2)$ & $*(4,4,3,2,2)$ \\
$*(4,2,3,3,3)$ & $(1,0,3,3,3)$ & $*(1,4,4,3,3)$ & $(0,0,4,3,3)$ \\
$(0,3,4,4,4)$ & $*(2,1,4,4,4)$ & $(2,0,0,4,4)$ & $(1,1,0,4,4)$ \\
$(a, b)=\left(\frac{2}{5}, \frac{2}{5}\right)$ & $(a, b)=\left(\frac{1}{5}, \frac{1}{5}\right)$ & $(a, b)=\left(\frac{1}{5}, \frac{2}{5}\right)$ & $(a, b)=\left(\frac{1}{5}, \frac{3}{5}\right)$ \\
\hline
\end{tabular}


In each column of the table there are two monomials that are distinguished by a $*$. These are monomials that have $\ell \neq 0$. Now it is clear that the relation (4.13) acts within each family and a little thought reveals that, on a family, the operator $\mathrm{e}^{-\pi \varphi Q}$ acts as a $5 \times 5$ matrix which, relative to a basis in which the three monomials with $\ell=0$ are taken first and the two with $\ell \neq 0$ are taken last, has the block structure

$$
\left(\begin{array}{ll}
1 & S \\
0 & G
\end{array}\right)
$$

where the 1 denotes a $3 \times 3$ matrix and $G$ denotes a $2 \times 2$ matrix. The unit matrix arises from the $j=0$ terms in (4.13) when $\ell=0$. Now the inverse of such a block matrix has a similar form, in fact

$$
\left(\begin{array}{cc}
\mathbf{1} & S \\
\mathbf{0} & G
\end{array}\right)^{-1}=\left(\begin{array}{cc}
\mathbf{1} & -S G^{-1} \\
\mathbf{0} & G^{-1}
\end{array}\right)
$$

The operator $\mathfrak{U}(0)$ will, in general, act between monomials from different families but the operator preserves the number of zeros of a vector of exponents. Thus the vectors that have $\ell \neq 0$ are mapped among themselves. By considering all the vectors together we can form big matrices for the operators $\mathrm{e}^{-\pi \varphi Q}$ and $\mathrm{e}^{\pi \varphi^{p} Q}$, but provided we write a basis with the vectors that have $\ell=0$ before those with $\ell \neq 0$ these big matrices will still take a block triangular form of the type we are discussing. In this basis, the operator $\mathfrak{U}(0)$ will be block-diagonal. It follows that the matrix $(\mathbf{1}-\mathfrak{U}(\varphi) T / p)$ is block upper triangular and hence its determinant reduces (apart from a term $(1-T / p)^{n}$, that is independent of $\varphi$ ) to the determinant of a matrix evaluated on the monomials that have $\ell \neq 0$.

If we evaluate the operator $\mathrm{e}^{-\pi \varphi Q}$ with respect to the bases provided by these vectors with $\ell \neq 0$ we find, on using (4.13) and the multiplication formula for the $\Gamma$-function in the form

$$
(5 m) !=5^{5 m} m !\left(\frac{1}{5}\right)_{m}\left(\frac{2}{5}\right)_{m}\left(\frac{3}{5}\right)_{m}\left(\frac{4}{5}\right)_{m},
$$

that it corresponds to a matrix that is essentially a Wronskian matrix of hypergeometric functions. Setting $z=\left(\frac{\varphi}{5}\right)^{5}$,

$$
\begin{aligned}
& f(a, b ; z)={ }_{2} F_{1}(a, b ; a+b ; z) \text { and } \\
& g(a, b ; z)=\frac{z^{1-a-b}}{1-a-b}{ }_{2} F_{1}(1-a, 1-b ; 2-a-b ; z)
\end{aligned}
$$


the matrix is

$$
G(a, b ; z)=\left(\begin{array}{ll}
f(a, b ; z) & z^{a+b} \frac{d}{d z} f(a, b ; z) \\
g(a, b ; z) & z^{a+b} \frac{d}{d z} g(a, b ; z)
\end{array}\right)
$$

where, for each family, the parameters $a, b$ take the values shown in table 2 . In each case, we have the relation

$$
\operatorname{det} G(a, b ; z)=\frac{1}{1-z}
$$

The values of the $a$ and $b$ coefficients given in the table differ from those that correspond to the $\mathcal{A}$ and $\mathcal{B}$ curves of [9] but this is due merely to the fact that here we have hypergeometric functions with argument $z$ while in [9] we were dealing with functions of argument $1 / z$. The functions

$$
z_{2}^{-a} F_{1}\left(a, 1-b ; 1+a-b ; \frac{1}{z}\right) \quad \text { and } \quad z_{2}^{-b} F_{1}\left(1-a, b ; 1-a+b ; \frac{1}{z}\right)
$$

satisfy the same differential equation as ${ }_{2} F_{1}(a, b ; a+b ; z)$.

The block structure of $\mathfrak{U}(0)$ depends on the value of $\rho$. We will illustrate the structure for $\rho=4$, since the other cases are simpler. The matrix $\mathfrak{U}(0)$ relates, for the case $\rho=4$, a vector $\mathbf{v}$ with the $\bmod 5$ reduction of $2 \mathbf{v}$. This process interchanges the permutations of the $(4,1,0,0,0)$ family with the $(3,2,0,0,0)$ family. We take as a basis the monomials:

$(2,1,4,4,4)$

$(3,4,1,1,1)$

$(1,2,4,4,4)$.

These are taken in pairs, alternately, from the $(4,1,0,0,0)$ and $(3,2,0,0,0)$ families and their permutations. With respect to this basis the matrix $\mathfrak{U}(\varphi)$ 
takes the form

$$
\left(\begin{array}{cccc}
\widetilde{G} & 0 & 0 & 0 \\
0 & \widetilde{H} & 0 & 0 \\
0 & 0 & \widetilde{G} & 0 \\
0 & 0 & 0 & \widetilde{H}
\end{array}\right)\left(\begin{array}{cccc}
0 & 0 & 0 & V \\
W & 0 & 0 & 0 \\
0 & V & 0 & 0 \\
0 & 0 & W & 0
\end{array}\right)\left(\begin{array}{cccc}
G & 0 & 0 & 0 \\
0 & H & 0 & 0 \\
0 & 0 & G & 0 \\
0 & 0 & 0 & H
\end{array}\right)=\left(\begin{array}{cccc}
0 & 0 & 0 & Y \\
Z & 0 & 0 & 0 \\
0 & Y & 0 & 0 \\
0 & 0 & Z & 0
\end{array}\right)
$$

where the blocks correspond to $2 \times 2$ matrices, $Y=\widetilde{G} V H$ and $Z=\widetilde{H} W G$. Thus the determinant we require takes the form

$$
\begin{aligned}
\operatorname{det}\left(\mathbf{1}-\mathfrak{U} \frac{T}{p}\right) & =\operatorname{det}\left(\begin{array}{cccc}
\mathbf{1} & 0 & 0 & -T Y / p \\
-T Z / p & \mathbf{1} & 0 & 0 \\
0 & -T Y / p & \mathbf{1} & 0 \\
0 & 0 & -T Z / p & \mathbf{1}
\end{array}\right) \\
& =\operatorname{det}\left(\mathbf{1}-\frac{T^{4}}{p^{4}}(Y Z)^{2}\right) .
\end{aligned}
$$

The last equality follows on factoring the matrix into the product of an upper triangular and lower triangular matrix

$$
\begin{aligned}
& \left(\begin{array}{cccc}
\mathbf{1} & 0 & 0 & -T Y / p \\
-T Z / p & \mathbf{1} & 0 & 0 \\
0 & -T Y / p & \mathbf{1} & 0 \\
0 & 0 & -T Z / p & \mathbf{1}
\end{array}\right) \\
& =\left(\begin{array}{cccc}
\mathbf{1} & -T^{3} Y Z Y / p^{3} & -T^{2} Y Z / p^{2} & -T Y / p \\
0 & \mathbf{1} & 0 & 0 \\
0 & 0 & \mathbf{1} & 0 \\
0 & 0 & 0 & \mathbf{1}
\end{array}\right)\left(\begin{array}{cccc}
\mathbf{1}-T^{4}(Y Z)^{2} / p^{4} & 0 & 0 & 0 \\
-T Z / p & \mathbf{1} & 0 & 0 \\
0 & -T Y / p & \mathbf{1} & 0 \\
0 & 0 & -T Z / p & \mathbf{1}
\end{array}\right) .
\end{aligned}
$$

It follows from these considerations that

$$
\operatorname{det}\left(\mathbf{1}-\mathfrak{U} \frac{T}{p}\right)=1+p^{6} c(\varphi) T^{4}+p^{12} T^{8}
$$


The explicit factors of $p$ follow from counting the explicit powers that appear in (4.12). The coefficient $c(\varphi)$ has, for $\varphi=\operatorname{Teich}(5) \psi$, a structure similar to that which we have seen in the previous section

$$
c(\varphi)=\frac{1}{p^{4}} \operatorname{Tr}\left((Y Z)^{2}\right)=\left(\frac{1-\psi^{5}}{1-\psi^{5 p^{n}}}\right) \tilde{c}_{n}(\operatorname{Teich}(5) \psi)+\mathcal{O}\left(p^{N}\right)
$$

which again allows us to recover the results of [9].

\section{Open problems}

We list here three open problems related to the present work.

\subsection{Special geometry}

A formulation of $p$-adic special geometry valid throughout the moduli space would seem to be a necessary prerequisite for a proper discussion of the arithmetic properties of conifolds and attractor geometries.

\subsection{The $\varphi=\infty$ limit}

Related to the above is the difficulty of developing a formalism analogous to Dwork analysis presented here but with the ability to expand the operator $\mathfrak{U}(\varphi)$ about $\varphi=\infty$ instead of expanding, as here, about $\varphi=0$. The variety corresponding to $\varphi=\infty$ is highly singular and this has, so far, prevented the application of the methods illustrated here. A specific question is what is the 'correct' form for the $\zeta$-function for the quintic for $\varphi=\infty$. In particular considerations of mirror symmetry [9] suggest that the 'correct' form of the factor $R_{\mathbf{1}}(\psi, T)$, for example, for $\psi=\infty$ should be

$$
(1-T)(1-p T)\left(1-p^{2} T\right)\left(1-p^{3} T\right)
$$

It is of interest to know if there is a reasonable way of justifying this form in terms of the action of the Frobenius map on the cohomology. When $\varphi=\infty$ the variety degenerates into five intersecting planes. Counting the $\mathbb{F}_{p}$-rational points is straightforward but does not give the desired form for the zeta-function. 


\subsection{Truncated periods versus infinite series}

In this work, we have calculated the numbers of points of the variety, say $N_{1}(\varphi)$, in terms of the periods, with the periods given by infinite series. In [6] these same numbers are calculated in terms of truncated periods and semiperiods, that is, periods and semiperiods truncated to the first $p$ terms whose argument is the parameter evaluated on the Teichmüller points. Since, in the latter approach, one deals with finite series there is no need for the process of analytic continuation that has occupied us here. It is of interest to understand how these two calculations are related. In particular, explicit expressions are given in [6] of the form

$$
N_{1}=\frac{1}{p-1}\left(p^{4}+\sum_{m=1}^{p-2} \beta_{m} \Lambda^{m}\right)
$$

with $\Lambda=1 /$ Teich $\varphi^{5}$ and the $\beta_{m}$ coefficients for which explicit expressions are known in terms of the $p$-adic $\Gamma$-function. It would be of interest to investigate whether similar expressions are available for the $\mathfrak{U}$ matrix evaluated on the Teichmüller points.

\section{Acknowledgment}

It is a pleasure to acknowledge fruitful conversations with Fernando Rodríguez-Villegas and Duco Van Straten and to thank Alan Lauder for much patient instruction.

\section{References}

[1] A. Weil, Solutions of equations in finite fields, Bull. Amer. Math. Soc. 55 (1949), 497-508.

[2] B. Dwork, On the rationality of the zeta function of an algebraic variety, Amer. J. Math. 82 (1960), 631-648.

[3] B. Dwork, On the zeta-function of a hypersurface, Publ. Math. I.H.É.S. 12 (1962), 5-68.

[4] B. Dwork, On the zeta-function of a hypersurface II, Ann. Math. 80 (1964), 227-299. 
[5] E. Frenkel, Lectures on the Langlands program and conformal field theory, $\S 1.2$, hep-th/0512172.

[6] P. Candelas, X. de la Ossa and F. Rodríguez-Villegas, Calabi-Yau manifolds over finite fields I, hep-th/0012233.

[7] N. Katz, Travaux de Dwork, Séminaire N. Bourbaki 409 (1971-1972), 167-200.

[8] N. Katz, On the differential equations satisfied by period matrices, Pub. Math. I.H.E.S. 35 (1969), 71-106.

[9] P. Candelas, X. de la Ossa and F. Rodríguez-Villegas, Calabi-Yau manifolds over finite fields II, in Calabi-Yau varieties and mirror symmetry, Toronto 2001, 121-157, hep-th/0402133.

[10] A. Lauder, Counting solutions to equations in many variables over finite fields, Found. Comput. Math. 4 (2004), 221-267.

[11] R. Gerkmann, Relative rigid cohomology and deformation of hypersurfaces, Int. Math. Res. Pap. (2007), Vol. 2007: article ID rpm 003, 67 p.

[12] N.M. Katz, Another look at the Dwork family, Preprint, 2007, available at http://www.math.princeton.edu/ nmk/.

[13] R. Kloosterman, The zeta-function of monomial deformations of Fermat hypersurfaces, ArXiv:math/0703120.

[14] B. Dwork, G. Gerotto and F.J. Sullivan, An Introduction to G-Functions, Annals of Mathematics Studies, 133, Princeton University Press, Princeton NJ, 1994.

[15] N. Koblitz, p-Adic numbers, p-adic analysis, and zeta functions, 2nd edn, Graduate Texts in Mathematics 58, Springer-Verlag, New York, 1984.

[16] M. van der Put, The cohomology of Monsky and Washnitzer, Mem. Soc. Math. France (NS) 23 (1986), 33-60.

[17] P. Monsky and G. Washnitzer, Formal cohomology. I., Ann. Math. 88(2) (1968), 181-217.

[18] P. Berthelot, Finitude et pureté cohomologique en cohomologie rigide, Invent. Math. 128 (1997), 329-377.

[19] A.M. Robert, The Gross-Koblitz formula revisited, Rend. Sem. Mat. Univ. Padova, 105 (2001), 157-170. 
[20] T.G. Abbott, K.S. Kedlaya and D. Roe, Bounding Picard numbers of surfaces using p-adic cohomology, to appear in Arithmetic, Geometry and Coding Theory, (AGCT 2005), Societé Mathématique de France.

[21] B. Dwork, p-Adic cycles, Publ. Math. I.H.É.S. 37 (1969), 27-115.

Mathematical Institute

OXFORD UNIVERSITY

24-29 St GiLes'

OXFORD OX1 3LB

$\mathrm{UK}$

E-mail address: candelas@maths.ox.ac.uk

E-mail address: delaossa@maths.ox.ac.uk

ReCeIVED May 15, 2007 